\title{
ELASTIC SOLUTIONS BASED ON THE MORI-TANAKA SCHEME FOR PRESSURIZED FUNCTIONALLY GRADED CYLINDER
}

\author{
Mehmet Eker ${ }^{1}$, Durmuş Yarımpabuç ${ }^{2}$, Ali Yıldırım ${ }^{3}$, Kerimcan Çelebi $^{4}$ \\ ${ }^{1}$ Department of Mechanical Engineering, Osmaniye Korkut Ata University \\ Osmaniye, Turkey \\ ${ }^{2}$ Department of Mathematics, Osmaniye Korkut Ata University \\ Osmaniye, Turkey \\ ${ }^{3}$ Osmaniye Vocational School, Osmaniye Korkut Ata University \\ Osmaniye, Turkey \\ ${ }^{4}$ Department of Mechanical Engineering, Ceyhan Faculty of Engineering, Çukurova University \\ Adana, Turkey \\ mehmeteker@osmaniye.edu.tr,durmusyarimpabuc@osmaniye.edu.tr, \\ aliyildirim@osmaniye.edu.tr,kcelebi@cu.edu.tr
}

Received: 11 August 2020; Accepted: 26 November 2020

\begin{abstract}
In this paper, an elastic analysis of a thick-walled functionally graded cylinder subjected to internal pressure is examined. Material properties for the isotropic material are estimated to obey the Mori-Tanaka homogenization scheme through the thickness. The resulting two-point irregular boundary value problem is solved by the pseudospectral Chebyshev method that converts the boundary value problem to the system of equations, which can be solved by any appropriate decomposition method. Benchmark solutions are used to validate the method. The effect of the arbitrarily chosen volume fraction index is demonstrated for stress and displacement distributions. The effective stresses for different inner radius and volume fraction index are also discussed.
\end{abstract}

MSC 2010: 74B05, 74E05, 74S25

Keywords: FGM, cylindrical pressure vessels, pseudospectral Chebyshev method, Mori-Tanaka homogenization

\section{Introduction}

Cylindrical elements are encountered in many structures such as containers, fittings, rolling mills, bearing supports, rods, pipes, tubes, pipelines, hydraulic systems, tin cans, etc. The cylinders as tubes are much easier to manufacture by using nineteenth-century machine tools due to their scalability, favorable surface-to-volume ratio, and ability to withstand pressure [1]. A new production aspect based on the graduation of at least two material components is proposed in the late twentieth century called functionally graded materials (FGM). The FGMs have a continuous change of properties from one point to another, making it possible to tailor 
desired mechanical properties. It is also very convenient to control the stresses by using graded materials in pressurized vessels. For these reasons, the elastic behavior of FGMs is a subject that is widely studied and continues to be investigated in the literature.

An exact analysis for FG pressure vessels is obtained Tütüncü and Öztürk [2]. Euler-Cauchy equations are solved in closed form for radial and circumferential stresses through the wall thickness of cylindrical and spherical vessels. Elastic analysis of exponentially graded cylindrical and spherical pressure vessels is carried out numerically by Chen et al. [3] and analytically by Eraslan and Akis [4]. Axisymmetric displacements and stresses in functionally graded hollow cylinders, disks, and spheres subjected to uniform internal pressure are obtained by Tütüncü and Temel [5] via the complementary functions method and plane elasticity theory. The transmission matrix method is defined as a numerical solution to calculate the displacement and stresses in FG cylinders and spheres in the study of Chen and Lin [6]. By assuming the displacement function is unknown and the elasticity modulus is arbitrary, an ordinary differential equation is derived. A technique is proposed by Nie et al. [7] for tailoring volume fractions of constituents in FG structures to attain either through-the-thickness uniform circumferential stress or in-plane shear stress. They tackled the rule of mixture and Mori-Tanaka scheme for homogenization in the radial direction. With the first-order shear deformation theory, Khoshgoftar et al. [8] studied a non-uniformly pressurized FG cylinder analytically. Xin et al. [9] solved the hypergeometric differential equation for the FG tube in terms of volume fractions of constituents. $\mathrm{Li}$ and $\mathrm{Liu}$ [10] considered nonaxisymmetric load for elastic behavior of FG cylinders with the assumption of arbitrary varying material properties. An exponentially graded cylindrical pressure vessel in the plane strain condition is analyzed by Nejad et al. [11]. The effect of the inhomogeneity on the stresses and different parameter values were considered, and the obtained results from the analytical solution were compared with the solution of the finite element method. Among the grading methods discussed so far, the Mori-Tanaka homogenization scheme has become prevalent in recent years for elastic analysis [12-14] or material tailoring [15] of FG pressure vessels. Therefore, this scheme is applied in an elastic analysis of FG cylindrical pressure vessels in this study.

In this paper, internally pressurized homogeneous and heterogeneous cylindrical pressure vessels were investigated utilizing the pseudospectral Chebyshev method. Displacement and stresses of the vessels are investigated for three types of vessels, and further discussion is made for different volume fraction indexes in the Mori-Tanaka case. What's more, the feasibility of the pseudospectral Chebyshev method in the solution of this type of problem is surveyed.

\section{Governing equations}

Consider thick-walled cylindrical pressure vessels subjected to internal pressure. The radial coordinate $\bar{r}$ and the displacement $\bar{u}$ are normalized with the outer 
radius of the cylinder $b$ such that $r=\bar{r} / b$ and $u=\bar{u} / b$. The material is isotropic and functionally graded. The axisymmetric governing equations to be presented in what follows apply to thick-walled hollow cylinders under the plane stress condition. Therefore, the strain-displacement and constitutive equations are [2]:

$$
\begin{gathered}
\varepsilon_{r}=\frac{d u}{d r}, \quad \varepsilon_{\theta}=\frac{u}{r} \\
\sigma_{r}=C_{11} \varepsilon_{r}+C_{12} \varepsilon_{\theta}, \quad \sigma_{\theta}=C_{12} \varepsilon_{r}+C_{11} \varepsilon_{\theta}
\end{gathered}
$$

where

$$
C_{11}=\frac{E(1-v)}{(1+v)(1-2 v)}, \quad C_{12}=\frac{E v}{(1+v)(1-2 v)} .
$$

The stress equilibrium equation is [2]

$$
\frac{d \sigma_{r}}{d r}+\frac{\sigma_{r}-\sigma_{\theta}}{r}=0
$$

with boundary conditions in radial stress as

$$
\sigma_{r}(a / b)=-P, \quad \sigma_{r}(1)=0
$$

where $P$ is the pressure applied on the inner surface of the cylinder, and $a$ is the inner radius. The outer surface is traction free. By using the strain-displacement and constitutive equations, an irregular linear ordinary differential equation (ODE) for radial displacement is obtained as:

$$
u^{\prime \prime}+S(r) u^{\prime}+Q(r) u=0
$$

with boundary conditions that can be obtained from constitutive equations of radial stress as:

$$
\left[C_{11} u^{\prime}+C_{12} \frac{u}{r}\right]_{r=a / b}=-P, \quad\left[C_{11} u^{\prime}+C_{12} \frac{u}{r}\right]_{r=1}=0
$$

Here ()$^{\prime}$ denotes the derivative concerning $\mathrm{r}$. The irregular variable coefficients $S(r)$ and $Q(r)$ are given in the Appendix for each case. Note that the derivative of the displacement is calculated by only multiplying it with the Chebyshev differentiation matrix, so no extra processing is required to calculate the stress values.

\subsection{Types of considered vessels}

In this study, three different types of cylinders are taken into consideration and compared in terms of their structural performances. One is homogeneous with a fully metal constituent and the other two are formed by functional grading of metal and ceramics. The radial grading of materials is done using both the power-law rule commonly used in the literature for possible analytical solutions and 
the Mori-Tanaka model. To comply with the analytical studies in the literature [2] in the power-law grading, the change of the elastic modulus in the radial direction is chosen as $E(r)=E_{c}(r / b)^{n}$, while the Poisson's ratio $\left(v=v_{m}\right)$ is accepted as constant. Here, $E_{c}$ is the elasticity modulus of the outer wall and $n$ is the inhomogeneity parameter. Note that $n=0$ corresponds to the homogeneous cylinder, and it should meet the value of the used real material couple as given in Table 1.

Table 1. Properties of constituent materials [16]

\begin{tabular}{|c|c|c|}
\hline Material/Properties & $E[\mathrm{GPa}]$ & $v$ \\
\hline$N i$ & 199.5 & 0.30 \\
\hline$S i_{3} N_{4}$ & 348.43 & 0.24 \\
\hline
\end{tabular}

Besides assuming the material properties vary according to a power-law function of $r$, the Mori-Tanaka homogenization scheme is inducted for determining the properties as the last case. The scheme is largely used to estimate elastic parameters of composite materials due to the eligibility of both dilute or high concentration of inclusion [12]. In the Mori-Tanaka method, the effective shear $(G)$ and bulk modulus $(K)$ are calculated as a function of the inclusions volume fraction. In this paper, to ensure the continuously graded compositional profile, the outer wall inclusion of the cylinder is assumed to follow the power-law volume fraction function in the radial coordinate as below [17]

$$
V_{c}=\left(\frac{r-a}{b-a}\right)^{\beta}, V_{m}=1-V_{c}
$$

where $\beta \geq 0$ is the volume fraction index and strongly affects the graduation of the composition. According to the current formulation, the inner surface is always metal and the outer surface is always ceramic. Therefore, this is not a general solution but a particular one for preferred material couples. Then, the effective local bulk and shear modulus included volume fraction function can be evaluated for the metal and ceramic constituents as [13]:

$$
\begin{gathered}
K_{m}=\frac{E_{m}}{3\left(1-2 v_{m}\right)}, \quad K_{c}=\frac{E_{c}}{3\left(1-2 v_{c}\right)} \\
G_{m}=\frac{E_{m}}{2\left(1+v_{m}\right)}, \quad G_{c}=\frac{E_{c}}{2\left(1+v_{c}\right)} .
\end{gathered}
$$

The effective modulus of bulk $(K)$ and shear $(G)$ are given by

$$
\frac{K-K_{m}}{K_{c}-K_{m}}=\frac{V_{c}}{1+V_{m}\left(\frac{K_{c}-K_{m}}{K_{m}+\frac{4}{3} G_{m}}\right)}, \frac{G-G_{m}}{G_{c}-G_{m}}=\frac{V_{c}}{1+V_{m}\left(\frac{G_{c}-G_{m}}{G_{m}+f_{1}}\right)}
$$

where $f_{1}=G_{m}\left(9 K_{m}+8 G_{m}\right) / 6\left(K_{m}+2 G_{m}\right)$. The effective modulus of elasticity $E$ and Poisson's ratio $v$ are determined by using the relation of [18] 


$$
E=\frac{9 K G}{3 K+G}, \quad v=\frac{3 K-2 G}{2(3 K+G)} .
$$

Here, subscript $c$ and $m$ represent ceramic and metal constituents, $a$ is the inner radius, and $b$ is the outer radius. For different values of $\beta$ the component distribution profiles of $E$ and $v$ along the radial direction are outlined in Figure 1. It can be seen that as the beta value decreases, the elastic modulus is upward concave, and as it increases, there is downward concave behavior. It is the opposite of Poisson's ratio. Besides, at the same position $(0.6<r<1)$ the modulus of elasticity increases as $\beta$ decreases, while for $v$ the reverse holds. It is clear that for $\beta=0$, it is a homogeneous ceramic material, and when it reaches the top, homogeneous metal will be obtained.
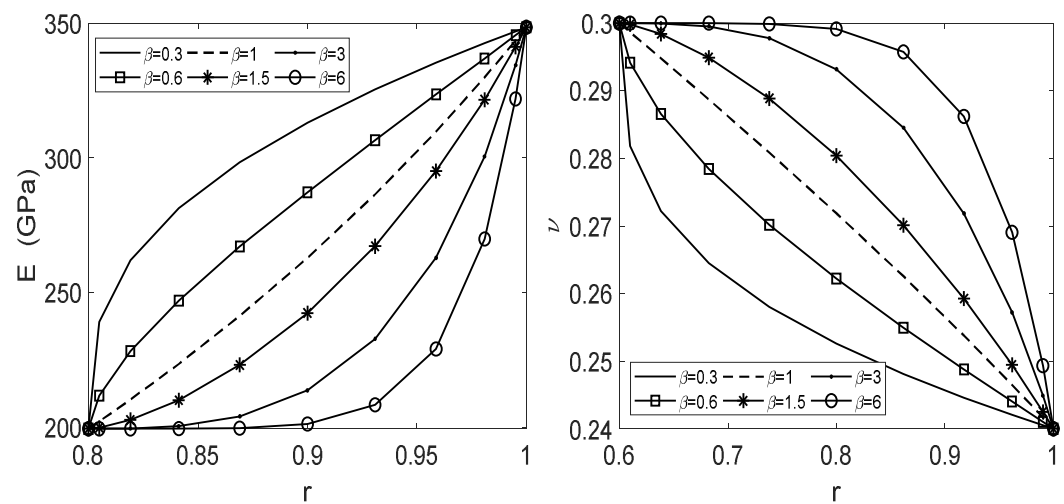

Fig. 1. Variations of modulus of elasticity and Poisson's ratio for different volume fraction index

\section{The pseudospectral Chebyshev method}

The Pseudospectral Chebyshev Method (Gottlieb [19], Fornberg [20], and Trefethen [21]), based on Chebyshev polynomials of the first kind, is used to perform the radial displacement and stress analysis of functionally graded hollow cylinders under the effect of pressure. Concerning collocation points, the first order $(N+1) x(N+1)$. The Chebyshev differentiation matrix

$$
0=r_{o}<\eta_{1} \ldots<r_{N} \text { with } r_{j}=\cos \left(\frac{j \pi}{N}\right), \quad(j=0,1, \ldots, N)
$$

will be denoted by $D$. The computation procedure of the Chebyshev differentiation matrix and codes as an m-file can be found in notable references, see e.g., [21], where the collocation points $r_{j}$ are numbered from right to left and defined in $[-1,1]$. With a small revision, the method can be implemented at any interval.

First-order Chebyshev differentiation matrix $D$ provides a highly precise approximation to $u^{\prime}\left(r_{j}\right), u^{\prime \prime}\left(r_{j}\right), \ldots$, simply by multiplying the differential matrix with 
vector data $u^{\prime}\left(r_{j}\right)=(D u)_{j}, u^{\prime \prime}\left(r_{j}\right)=\left(D^{2} u\right)_{j}$, such like where $u=\left[u_{0}, \ldots, u_{N}\right]^{T}$ discrete vector data at positions $r_{j}$. The detailed implementation of the method is explained in the study of Trefethen [21]. Therefore, the linear axisymmetric displacement equation for the cylinder (9) is simply converted into a linear system by using the pseudospectral Chebyshev collocation method as follows:

$$
M_{u} u=0
$$

where

$$
M_{u}=D^{2}+S(r) D+Q(r) .
$$

Boundary conditions (10) are imposed on this linear system (18) by only replacing the first and last row of the system matrix $M_{u}$ with the appropriate values. Then, the nondimensional displacement can be found by solving the linear system (18) by any decomposition method.

\section{Numerical results}

The results of stresses and displacement are presented for $a / b=0.6$ and 0.8 . In the homogeneous case, the cylinder is assumed fully metal. In power-law grading, the Poisson's ratio is kept constant as $v_{m}=0.3$. For a meaningful comparison of homogeneous, power-law, and Mori-Tanaka vessels, inhomogeneity parameters are calculated as $n=1.091611$ and its corresponding volume fraction index for Mori-Tanaka is $\beta=0.814131$ for $a / b=0.6$ vessel and similarly, $n=2.49894$ and its corresponding $\beta=0.907125$ for the vessel of $a / b=0.8$. For comparison, while the inhomogeneous parameters and volume fraction indexes are taken with these values for the distribution of Young's modulus, Poisson ratios are kept constants $\left(v=v_{m}\right)$. In Mori-Tanaka homogenization, both the Young modulus and Poisson's ratio vary through the wall thickness. The radial variation of elastic properties is shown in Figure 1 for different index values. The cylinder is experiencing $P_{i}=50 \mathrm{MPa}$ internal pressure in all cases. The stresses are nondimensionalized by corresponding applied internal pressure, and the deformations are nondimensionalized by outer radii $b$.

The verity of the pseudospectral Chebyshev (PSD) method is first checked with a well-known stress expression (found in [2]) given below for a homogeneous cylinder under internal pressure $P$ and presented in Table 2. Further comparison is made for the radial and hoop stresses of the power-law graded vessel in Table 3. It can be noted from the tables that the PCM results match quite well (seven-digit accuracy for $N=11$ ) with the analytical results that demonstrate the validity of the numerical solutions used in this work [2]. The stresses for the homogeneous vessel can be calculated from [2] or [22].

$$
\sigma_{r}^{H}=\frac{P a^{2}}{b^{2}-a^{2}}\left(1-\frac{b^{2}}{r^{2}}\right), \quad \sigma_{\theta}^{H}=\frac{P a^{2}}{b^{2}-a^{2}}\left(1+\frac{b^{2}}{r^{2}}\right)
$$


Table 2. Comparison of PCM with an analytical solution for homogeneous cylindrical pressure vessel [2]

\begin{tabular}{|c|c|c|c|c|}
\hline & \multicolumn{2}{|c|}{ Radial Stress } & \multicolumn{2}{c|}{ Hoop Stress } \\
\hline $\mathrm{r}$ & Analytic & Numeric & Analytic & Numeric \\
\hline 0.600000000 & -1.000000000 & -1 & 2.125000000 & 2.125000041 \\
\hline 0.609788696 & -0.950238245 & -0.950238348 & 2.075238245 & 2.075238240 \\
\hline 0.638196601 & -0.818563199 & -0.818563198 & 1.943563199 & 1.943563234 \\
\hline 0.682442949 & -0.645285535 & -0.645285625 & 1.770285535 & 1.770285526 \\
\hline 0.738196601 & -0.469734676 & -0.469734674 & 1.594734676 & 1.594734700 \\
\hline 0.800000000 & -0.316406250 & -0.316406325 & 1.441406250 & 1.441406236 \\
\hline 0.861803398 & -0.194866552 & -0.194866551 & 1.319866552 & 1.319866567 \\
\hline 0.917557050 & -0.105622924 & -0.105622989 & 1.230622924 & 1.230622908 \\
\hline 0.961803398 & -0.045564867 & -0.045564867 & 1.170564867 & 1.170564877 \\
\hline 0.990211303 & -0.011176114 & -0.011176174 & 1.136176114 & 1.136176097 \\
\hline 1.000000000 & 0.000000000 & 0.000000000 & 1.125000000 & 1.125000008 \\
\hline
\end{tabular}

Table 3. Comparison of PCM with an analytical solution for power-law grading cylindrical pressure vessel [2]

\begin{tabular}{|c|c|c|c|c|}
\hline & \multicolumn{2}{|c|}{ Radial Stress } & \multicolumn{2}{c|}{ Hoop Stress } \\
\hline $\mathrm{r}$ & Analytic & Numeric & Analytic & Numeric \\
\hline 0.600000000 & -1.000000000 & -0.999999999 & 1.361758273 & 1.361758154 \\
\hline 0.609788696 & -0.962044759 & -0.962045009 & 1.367128733 & 1.367128505 \\
\hline 0.638196601 & -0.858004069 & -0.858004069 & 1.383644652 & 1.383644527 \\
\hline 0.682442949 & -0.711770512 & -0.711770775 & 1.411702504 & 1.411702258 \\
\hline 0.738196601 & -0.549956614 & -0.549956612 & 1.450198762 & 1.450198620 \\
\hline 0.800000000 & -0.393689120 & -0.393689404 & 1.495867608 & 1.495867334 \\
\hline 0.861803398 & -0.256474708 & -0.256474707 & 1.543774893 & 1.543774731 \\
\hline 0.917557050 & -0.145736899 & -0.145737204 & 1.588356503 & 1.588356202 \\
\hline 0.961803398 & -0.065135176 & -0.065135175 & 1.624408243 & 1.624408066 \\
\hline 0.990211303 & -0.016329231 & -0.016329548 & 1.647796487 & 1.647796170 \\
\hline 1.000000000 & 0.000000000 & 0.000000000 & 1.655892318 & 1.655892136 \\
\hline
\end{tabular}

The numerical results based on the three different compositional cylinders with the boundary conditions (Eqs. (10)) for radial displacement and radial and hoop stresses are plotted in Figure 2 for different aspect ratio $a / b$. The radial displacement values decrease slightly from the inner surface through the outer wall for all cases in Figure 2a-b. The variation in the displacement of power-law graded vessel is similar to that of homogenous material. However, the displacement of the Mori-Tanaka based model is less than the others. The comparative normalized radial 
stresses are plotted in Figure 2c-d. For all compositional cases, the magnitude of the compressive radial stress has a monotonic behavior across the wall thickness. It can be seen that for any point in $a<r<b$, the Mori-Tanaka cylinder has the lowest radial stress magnitude for both ratios $a / b$. Due to the normalization, $\sigma_{r}=-1$ at $r=a$ and $\sigma_{r}=0$ at $r=b$. The tensile hoop stress distributions across the normalized radial direction for each case is given in Figure 2e-f. The results show that hoop stress nearly stays stable for the ratio of $a / b=0.6$ and tents to increase along the outer wall if the thickness gets thinner except for the homogeneous case. Among the three different compositions, the Mori-Tanaka scheme supplies the lowest and more stationary results for dimensionless hoop stress $\left(\sigma_{\theta}\right)$. Overall, the increase of displacement and hoop stress values occur proportionally to $a / b$. In other words, when the wall thickness is halved, the displacement and hoop stress values nearly double up. It can also be seen that the Mori-Tanaka homogenization scheme behaves quite differently from the others.

After a comparison of different material composition, the Mori-Tanaka based pressure vessel of wall thickness $a / b=0.8$ subjected to internal pressure is taken into consideration for further investigation. Computations are performed for different values of the volume fraction index, such as $\beta=0.6,1.5,3$. The results of these calculations are depicted in Figure 3. The variation of nondimensional displacement through the radial direction based on the Mori-Tanaka model is shown in Figure 3a. According to these figures, by increasing $\beta$, which means the major of stiffness similar to the metal constituent, the value of radial displacement increases. Choosing lower $\beta$ keeps stiff distribution near the outer side and offers the lower radial displacement. The volume fraction index $\beta$ can significantly affect the stress distribution along the radius direction.

For radial stress in Figure 3b, although the trend is similar, in any point of radial coordinate, the lowest radial stress comes out with a lower $\beta$ value. For hoop stress, the influence of $\beta$ is completely different in Figure $3 \mathrm{c}$. At the smaller index value, the hoop stress has the lowest amount, in addition to this it increases relatively faster near the inner wall and it almost keeps its value around the outer wall. By the increase of fraction index, the hoop stress increases at the inner and outer walls but the fact remains that it supplies lower magnitude around the middle of the wall thickness. The variation of the fraction index has some effects on the distribution of the hoop stress.

The effect of wall thickness ratio $(a / b)$ and volume fraction coefficients are investigated for equivalent stress with the help of von-Mises criteria (Eq. (21)). As Table 4 shows, the maximum equivalent stress of the vessel occurs in a lower wall thickness ratio and larger amounts of $\beta$. The inverse, corresponds to safer situation. Further scrutiny reveals that if the $a / b$ increases, the equivalent stress variation decreases with the increase of $\beta$.

$$
\sigma_{e f f}=\left\{\frac{1}{2}\left[\left(\sigma_{1}-\sigma_{2}\right)^{2}+\left(\sigma_{2}-\sigma_{3}\right)^{2}+\left(\sigma_{3}-\sigma_{1}\right)^{2}\right]\right\}^{1 / 2}
$$




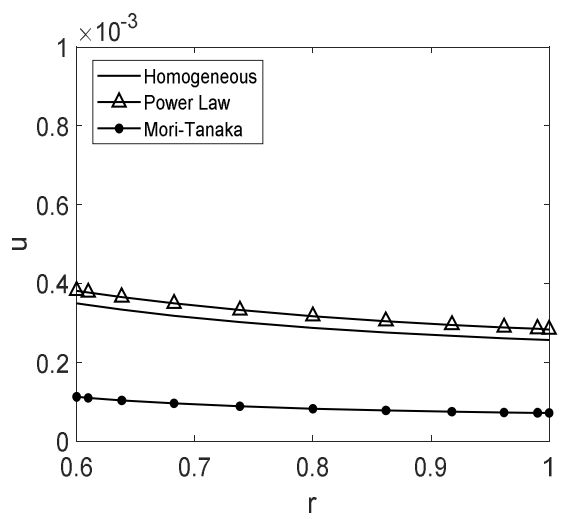

(a)

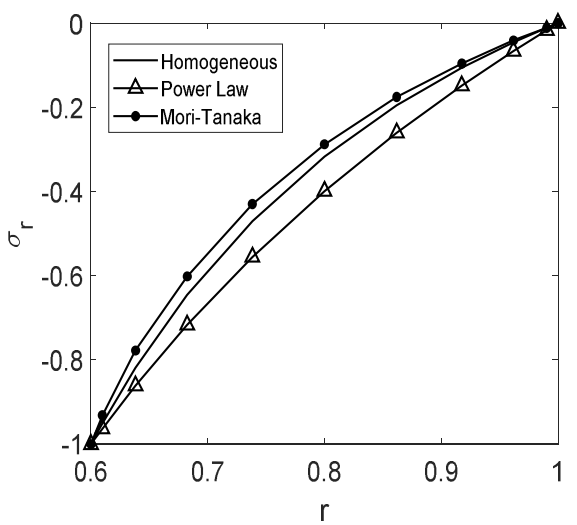

(c)

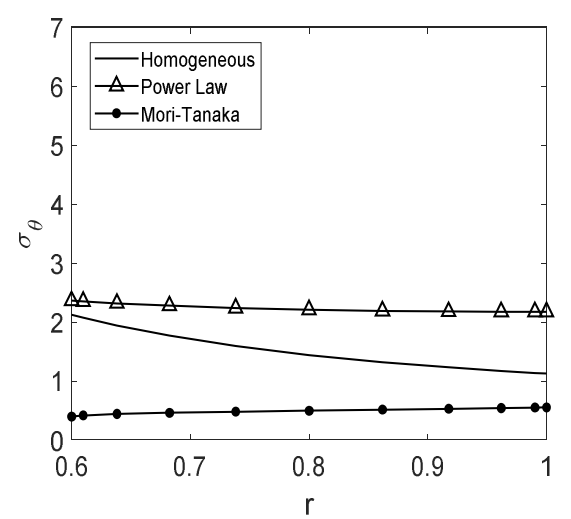

(e)

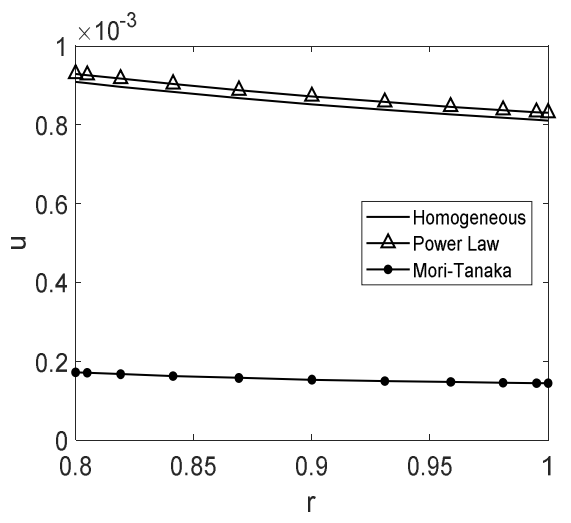

(b)

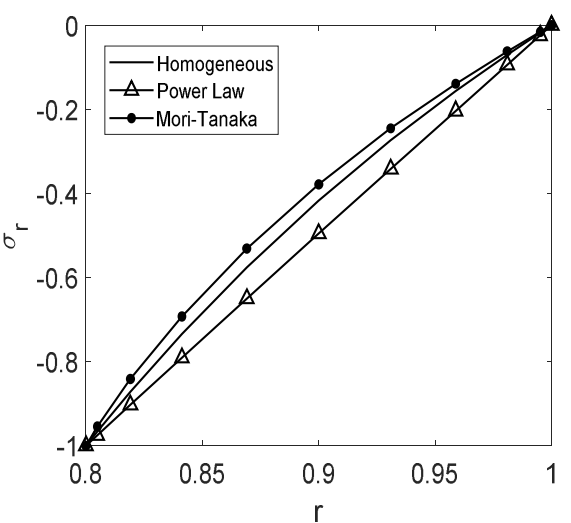

(d)

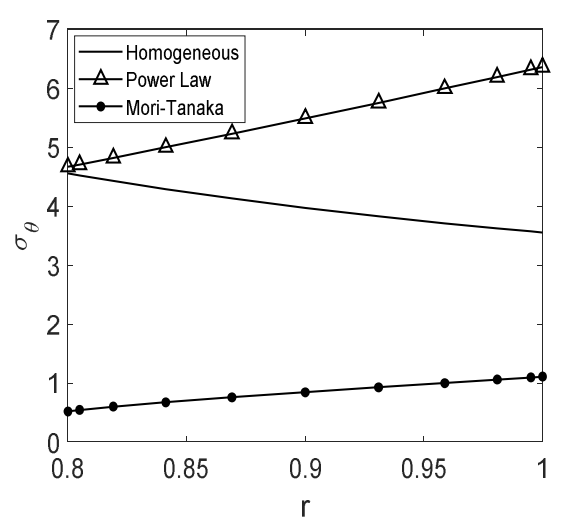

(f)

Fig. 2. Radial displacement and stresses for different thick-walled cylinders (left column for $a / b=0.6$, right column for $a / b=0.8$ ) 


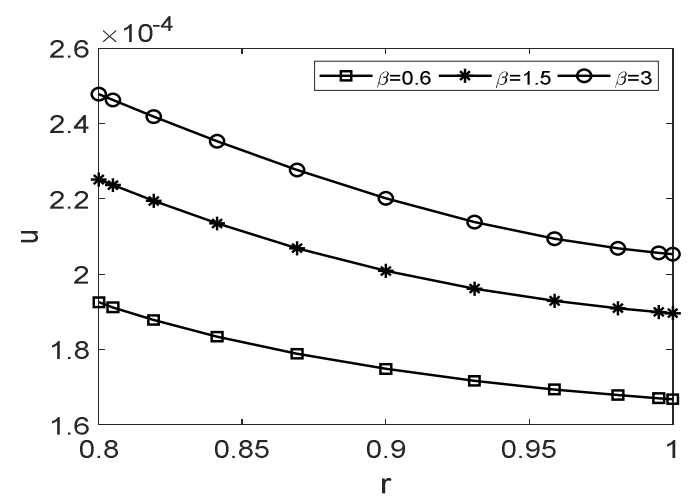

(a)

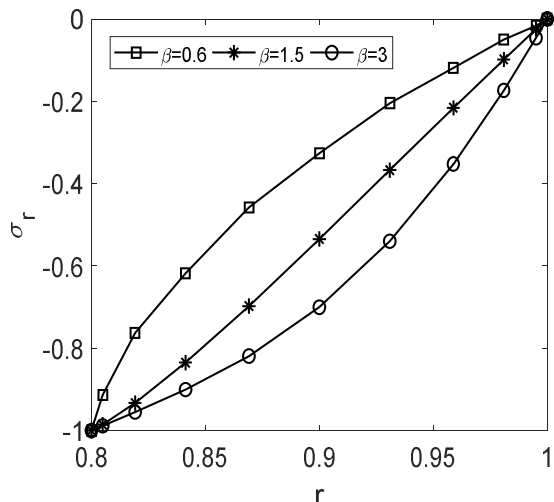

(b)

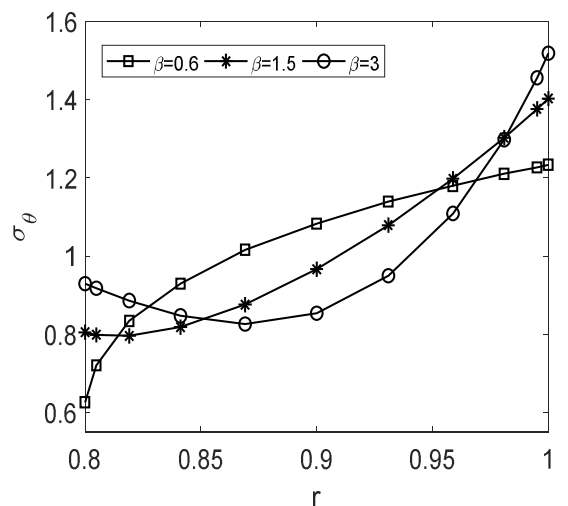

(c)

Fig. 3. Radial displacement and stresses of FG cylinder for Mori-Tanaka based different volume fraction index

Table 4. Comparison of effective stresses [MPa] for Mori-Tanaka cylinder with different inner radius and volume fraction index

\begin{tabular}{|c|c|c|c|}
\hline$a / b$ & $\beta=0.6$ & $\beta=1.5$ & $\beta=3$ \\
\hline 0.5 & 72.867 & 83.718 & 91.536 \\
\hline 0.6 & 74.156 & 84.205 & 91.774 \\
\hline 0.7 & 77.038 & 86.292 & 93.305 \\
\hline 0.8 & 82.396 & 90.526 & 96.516 \\
\hline 0.9 & 111.040 & 119.807 & 125.610 \\
\hline
\end{tabular}

\section{Conclusions}

In this paper, an elastic analysis of a thick-walled functionally graded cylinder subjected to constant pressure is developed by the pseudospectral Chebyshev 
method. It is observed that Mori-Tanaka homogenization gives better results compared to the gradings commonly used in the literature. To show the compositional difference in the elastic analysis, different values are considered for the volume fraction index. It has been found that the hoop stress is highly dependent on the composition of the materials and can be regulated with the appropriate index value. The derived results indicate that the volume fraction index has significant influence on the elastic property distribution of FG cylindrical vessels and can be optimized according to the required performance. Comparing the results of the literature in the form of a table, the proposed numerical method has splendid accuracy, so it is practical and easy to apply to such problems.

\section{References}

[1] Müller-Sievers, H. (2012). The Cylinder: Kinematics of the Nineteenth Century, 9. University of California Press.

[2] Tütüncü, N., \& Öztürk, M. (2001). Exact solutions for stresses in functionally graded pressure vessels. Composites Part B: Engineering, 32(8), 683-686. DOI: 10.1016/s1359-8368(01)00041-5.

[3] Chen, Y.Z., Lin, X.Y., Zhang, J.J., \& You, X.Y. (2008). Elastic analysis for thick cylinders and spherical pressure vessels made of functionally graded materials. Computational Materials Science, 44(2), 581-587. DOI:10.1016/j.commatsci.2008.04.018.

[4] Eraslan, A.N., \& Akış, T. (2015). Analytical solutions to elastic functionally graded cylindrical and spherical pressure vessels. Journal of Multidisciplinary Engineering Science and Technology, 2(10), 2687-2693.

[5] Tütüncü, N., \& Temel, B. (2009). A novel approach to stress analysis of pressurized FGM cylinders, disks and spheres. Composite Structures, 91(3), 385-390. DOI:10.1016/j.compstruct. 2009.06.009.

[6] Chen, Y.Z., \& Lin, X.Y. (2010). An alternative numerical solution of thick-walled cylinders and spheres made of functionally graded materials. Computational Materials Science, 48(3), 640-647. DOI:10.1016/j.commatsci.2010.02.033.

[7] Nie, G.J., Zhong, Z., \& Batra, R.C. (2011). Material tailoring for functionally graded hollow cylinders and spheres. Composites Science and Technology, 71(5), 666-673. DOI:10.1016/ j.compscitech.2011.01.009.

[8] Khoshgoftar, M.J., Rahimi, G.H., \& Arefi, M. (2013). Exact solution of functionally graded thick cylinder with finite length under longitudinally non-uniform pressure. Mechanics Research Communications, 51, 61-66. DOI:10.1016/j.mechrescom.2013.05.001.

[9] Xin, L., Dui, G., Yang, S., \& Zhang, J. (2014). An elasticity solution for functionally graded thick-walled tube subjected to internal pressure. International Journal of Mechanical Sciences, 89, 344-349.

[10] Li, H., \& Liu, Y. (2014). Functionally graded hollow cylinders with arbitrary varying material properties under nonaxisymmetric loads. Mechanics Research Communications, 55, 1-9.

[11] Nejad, M.Z., Abedi, M., Lotfian, M.H., \& Ghannad, M. (2016). Exact and numerical elastic analysis for the FGM thick-walled cylindrical pressure vessels with exponentially-varying properties. Archives of Metallurgy and Materials, 61(3), 1649-1654.

[12] Xin, L., Yang, S., Zhou, D., \& Dui, G. (2016). An approximate analytical solution based on the Mori-Tanaka method for functionally graded thick-walled tube subjected to internal pressure. Composite Structures, 135, 74-82. 
[13] Temel, B., Yildirim, S., \& Tutuncu, N. (2014). Elastic and viscoelastic response of heterogeneous annular structures under arbitrary transient pressure. International Journal of Mechanical Sciences, 89, 78-83.

[14] Najibi, A., \& Shojaeefard, M.H. (2016). Elastic mechanical stress analysis in a 2D-FGM thick finite-length hollow cylinder with newly developed material model. Acta Mechanica Solida Sinica, 29(2), 178-191.

[15] Dehnavi, F.N., Parvizi, A., \& Abrinia, K. (2018). Novel material tailoring method for internally pressurized FG spherical and cylindrical vessels. Acta Mechanica Sinica, 34(5), 936-948.

[16] Yıldırım, V. (2017). Heat-induced, pressure-induced and centrifugal-force-induced exact axisymmetric thermo-mechanical analyses in a thick-walled spherical vessel, an infinite cylindrical vessel, and a uniform disk made of an isotropic and homogeneous material. International Journal of Engineering \& Applied Sciences (IJEAS), 9(2), 66-87.

[17] Shariyat, M. (2012). Nonlinear transient stress and wave propagation analyses of the FGM thick cylinders, employing a unified generalized thermoelasticity theory. International Journal of Mechanical Sciences, 65(1), 24-37.

[18] Dai, H.L., Rao, Y.N., \& Dai, T. (2016). A review of recent researches on FGM cylindrical structures under coupled physical interactions, 2000-2015. Composite Structures, 152, 199-225.

[19] Gottlieb, D. (1981). The stability of pseudospectral-Chebyshev methods. Mathematics of Computation, 36(153), 107-118.

[20] Fornberg, B. (1998). A Practical Guide to Pseudospectral Methods, 1. Cambridge: Cambridge University Press.

[21] Trefethen, L.N. (2000). Spectral Methods in Matlab, 10, SIAM, Philadelphia, PA.

[22] Timoshenko, S.P, \& Goodier, J.N. (1970). Theory of Elasticity, Third Edition. New York: McGraw-Hill.

\section{Appendix}

The irregular variable coefficients of the linear ordinary differential equation for radial displacement are given below for all cases:

\section{Homogeneous case:}

$$
S(r)=\frac{1}{r}, \quad Q(r)=-\frac{1}{r^{2}}
$$

\section{Power-Law case:}

$$
S(r)=\left[2+\beta+\frac{1}{(v-1)}\right] \frac{1}{r}, \quad Q(r)=\left[\frac{1-(\beta+1) v}{(v-1)}\right] \frac{1}{r^{2}}
$$

\section{Mori-Tanaka case:}

$$
S(r)=\frac{C_{11}^{\prime}}{C_{11}}+\frac{1}{r}, \quad Q(r)=\frac{C_{11}^{\prime}}{C_{11}} \frac{1}{r}-\frac{1}{r^{2}} .
$$

